\title{
Electric Field and Energy Losses of Rounded Superconducting / Ferromagnetic Heterostructures at Self-Field Conditions
}

\author{
M. U. Fareed, B. C. Robert, and H. S. Ruiz
}

\begin{abstract}
The AC losses induced by an alternating transport current in type-II superconductors is a well known phenomena which still attracts much attention due to its intrinsic relevance for the proper development of practical applications. In the case of single core superconducting cables of cylindrical crosssection, it is possible to find exact analytical solutions at self field conditions, and it has been believed for nearly two decades that the use of an ideal soft ferromagnetic sheath with negligible magnetization losses will not affect the electromagnetic properties of the superconducting wire, and on the contrary due to the shielding magnetic properties of the ferromagnet, the total AC losses of the SC wire have to be reduced or as maximum they must be equal to the one for the bare superconductor at self-field conditions, what contraries the experimental evidences that show a non-negligible increase on the AC losses. In this paper, we explain the physical nature of this mysterious increase on the AC losses for rounded superconducting/ferromagnetic heterostructures, which for the sake of generality, it has been solved within the critical state theory and, a magnetic multipolar expansion which enables the direct coupling of the magnetostatic properties of the superconductor and an ideal soft ferromagnet. A significant increase on the transient electric field during the excitation period has been observed, which might have utter implications on the adequate choosing of insulation materials for superconducting/ferromagnetic heterostructures.
\end{abstract}

Index Terms-AC losses, Soft ferromagnets, Superconducting Ferromagnetic heterostructures, Magnetic multipoles.

\section{INTRODUCTION}

$\mathbf{M}$ ACROSCOPIC Superconducting/Ferromagnetic heterostructures have been the focus of considerable attention in recent years due to the novel phenomena and applications that can be envisaged with their use [1] $-[9]$. Particular focus has been played to the role of magnetization and demagnetization properties of these systems [9], [10], magnetic cloaking features [4]-[7], [11], and shielding properties of type-II superconductors (SC) surrounded or in the near proximity of a soft ferromagnetic material (FM) [12]-[21].

Manuscript submitted January 21, 2019.

The authors are with the Department of Engineering and the Leicester Institute for Space \& Earth Observation Science of the University of Leicester, Leicester, LE17RH, U.K. (e-mail: dr.harold.ruiz@leicester.ac.uk)

H. S. Ruiz acknowledges the use of the High Pertormance Computing Cluster Facilities (ALICE) provided by the University of Leicester, and the funding support provided by the EPSRC and the East Midlands Energy Research Accelerator ERA. M. U. Fareed thanks the College of Science and Engineering Scholarship Unit of the University of Leicester. B. C. Robert thanks the Scholarship unit of the Niger Delta Development Commission.

Color versions of one or more of the figures in this paper are available online at http://ieeexplore.ieee.org

Digital Object Identther XXXXX
However, the influence of the physical coupling between the macroscopic electromagnetic properties of these two systems on the overall hysteresis losses of the SCFM heterostructures for $\mathrm{AC}$ applications, is yet to be understood.

The fundamental ansatz behind these studies lies on the fact that a FM aid to amend the SC performance by shielding it from the self-induced magnetic field in multi-filament/layer compounds subjected to a transport current, as well as of any externally imposed magnetic field to the SC [22]. Therefore, as the critical current density in most of the type-II SCs is degraded by increasing the magnitude of the applied magnetic field, $H_{a}$, when the SC is encompassed within or in a near proximity of a FM, the SC exhibit enlarged critical currents through wide ranges of intensities of $H_{a}$. Because of this, the magnetic shielding benefits of SCFM heterostructures have been broadly reported on type-II SC cables of cylindrical cross-section composed by NbTi [12], BPSCCO [13], [14], $\mathrm{BSCCO}$ [15], YBCO [19], and $\mathrm{MgB}_{2}$ [16]-[18], as well as in other SC topologies (strips and bulks) [19]-[21]. However, a crucial question here is whether the presence of a FM will affect the AC losses of the SC in conditions where only applied current is considered (self-field conditions). Intuitively, we could think that the use of a sheath made of an ideal FM does not have any effect on the AC losses of a SC, as long as there is not any chemical driven reaction which could add superficial eddy currents at the interface between the two materials, as by definition a soft magnetic material with constant magnetic permeability produces null magnetic hysteresis losses. Nevertheless, a non negligible increase in the AC losses of these systems has been already reported for multiple SCFM configurations either under external magnetic field conditions (no transport current) [23], or in the absence of an applied magnetic field but with the presence of a transport current [23]-[25], which does not match with the losses provided by the bare FM at low field frequencies comparable with the self-field magnitude [26].

In the case of a SC strip of thickness $d_{S C}$ deposited over a thin FM layer, it has been demonstrated by simplified semianalytical methods that the critical state (CS) model allows to reproduce the increment of their $\mathrm{AC}$ losses at low magnetic fields $\left(H_{a}<0.14 J_{c} d_{S C}\right)$ applied perpendicularly to the surface of the heterostructure, as well as its $\mathrm{AC}$ loss reduction at greater strengths of the field (compared to the bare SC strip) up to reaching the saturation of the FM layer, when the losses of the SCFM heterostructure coincide with the curve of losses for the bare SC [27]. However, with the use of more sophisticated 

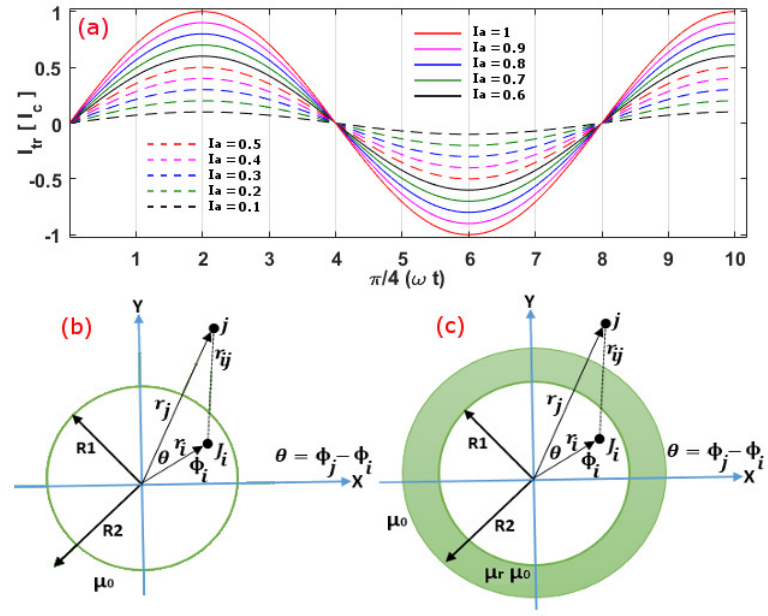

Figure 1. (Color online) Schematic plot of (a) the applied transport current $I_{t r}=I_{a} \sin \omega t$ in $I_{c}$ units for (b) a SC rounded wire of radius $R_{1}$ which is then (c) embedded within a finite FM sheath of outer radius $R_{2}$ and relative magnetic permeability $\mu_{r}$. Ten different amplitudes $I_{a}$ are considered for the AC losses calculations in both cases, the SC and SCFM heterostructure.

numerical approaches [28], [29], which indeed are extensively used for reproducing the electromagnetic properties of (bare) type-II SCs under many different configurations, for the case of SCFM heterostructures, it has been demonstrated that even for an infinitely thin SC strip, the reduction of the AC losses at low magnetic fields cannot be reproduced within or beyond the CS model. Therefore, some inductive coupling happens between the elements of current inside the SC specimen and the FM itself which cannot be incorporated within 1D systems, and in consequence it need to be directly included within the physical formalism of the problem in 2D and 3D symmetries.

Similar behaviour has been found for the case of rounded SCFM heterostructures under applied magnetic field, with the AC losses behaviour explained for moderate to high magnetic fields, it relative to the full penetration field $\left(H_{p}=2 \pi J_{c} R_{S C}\right)$ of the bare $\mathrm{SC}$ of radius $R_{1}$ [30]. However, a physical explanation for the increment in the AC losses of SCFM heterostructures at self-field conditions, i.e., with an applied transport current but no external magnetic field, has not been reached yet. In fact, for nearly two decades it has been believed that for isotropic type-II SCs of circular cross section, nor a $J_{c}$ degradation neither an increase in the AC losses can be caused by a FM sheath. The first statement being in full agreement with the CS model where a constant $J_{c}$ is customarily used for the modelling of the physical properties in practical superconductors [28], [31], but the latter not being justified by the simple use of the CS material law $\left(J \leq J_{c}\right)$, as the magneto-coupling between the elements with critical current density inside the SC and the magnetic properties of the FM is not explicitly included into the mathematical formulation that is used to describe this phenomena.

Thus, in Section III we present a semi-analytical $A-J$ formulation which allows the integral solution of a simplified minimization functional for the critical state solution of finite rounded SCFM heterostructures, with an explicit inductive coupling between the SC and FM specimens. Then, in Sec- tion [II we show some of our obtained results when an ideal FM sheath of relative magnetic permeability $\mu_{r}=46$ (a common value for Fe-based FMs) and outer radius $R_{2}=1.5 R_{1}$ is considered, providing a solid physical explanation to the origin of the increment in the AC losses of SCFM rounded heterostructures at self-field conditions. Finally, Section IV] is devoted to present the main conclusions of this study.

\section{Electromagnetic Formulation FOR ROUNDED SCFM HETEROSTRUCTURES}

In this section, the theoretical framework that enables a straightforward inclusion of the magnetostatic coupling between a cylindrical SC and a finite FM sheath (Figure 11) is disclosed. Our formalism is based upon the CS theory and the integral formalism developed at [28], where a generalized minimization functional can be obtained within the Magneto Quasi Steady approach $\left(\partial_{t} D \simeq 0\right)$ for decoupling the electric from the magnetic sectors at a local level, i.e., to enable the solution of the entire problem by only calculating the elements of current inside inside the SC domains. Thus, in general terms the functional to be minimized is defined as

$$
\mathcal{F}[\mathbf{B}(\cdot)]=\operatorname{Min} \int_{\Re^{3}} \frac{1}{2}|\Delta \mathbf{B}|^{2},
$$

which is equivalent to the energy minimization principle [32]

$$
\mathcal{F}\left[\mathbf{J}_{l+1}\right]=\operatorname{Min}\left\{-\int_{\Re^{3}} \mathbf{E} \cdot \mathbf{J}_{l+1}\right\},
$$

as long as the minimisation process accomplishes the constraint conditions enforced by the given sources and, the CS material law within the domains with current density, $\mathbf{J} \in \cdot \Delta_{r}$. Therefore, if the SC is carrying a transport current $I_{t r}$ flowing across the surface $\Omega$, it is mandatory to consider the extrinsic constraint

$$
\int_{\Omega} \mathbf{J} \cdot \hat{\mathbf{n}} d \Omega=I_{t r}
$$

which updates the distributions of local current to fulfil the physical condition $\mathbf{E} \cdot \mathbf{J}$ in those regions where the magnetic flux does not vary, being this consistent with the charge conservation principle for type-II SCs [33].

Thus, In order to deal with the coupling between a SC wire and a finite FM sheath, both of cylindrical cross section, these minimization functionals can be reduced to a $2 \mathrm{D}$ system, as long as it is assumed that the length of the wire is much greater than its radius, such that flux cutting effects at its terminals can be neglected [34], [35]. In this sense, assuming that the SC and the FM sheath are infinitely long along the z-axis, the resulting vector potential does not have components along the polar and radial directions, and the minimization can be only satisfied if the gradient of the scalar electric potential is equal to a time-dependent constant, $\nabla \phi=C_{t} \hat{u}_{z}$, such that the minimization functional can be reduced to

$$
\mathcal{F}=\operatorname{Min} \int_{\Omega}\left[\Delta \mathbf{A}_{z} \cdot \mathbf{J}+C_{t} J \Delta t\right],
$$

where $\Omega$ denotes the cross-section area of the SC, as the FM is assumed to be either a non-conductive material or one with 

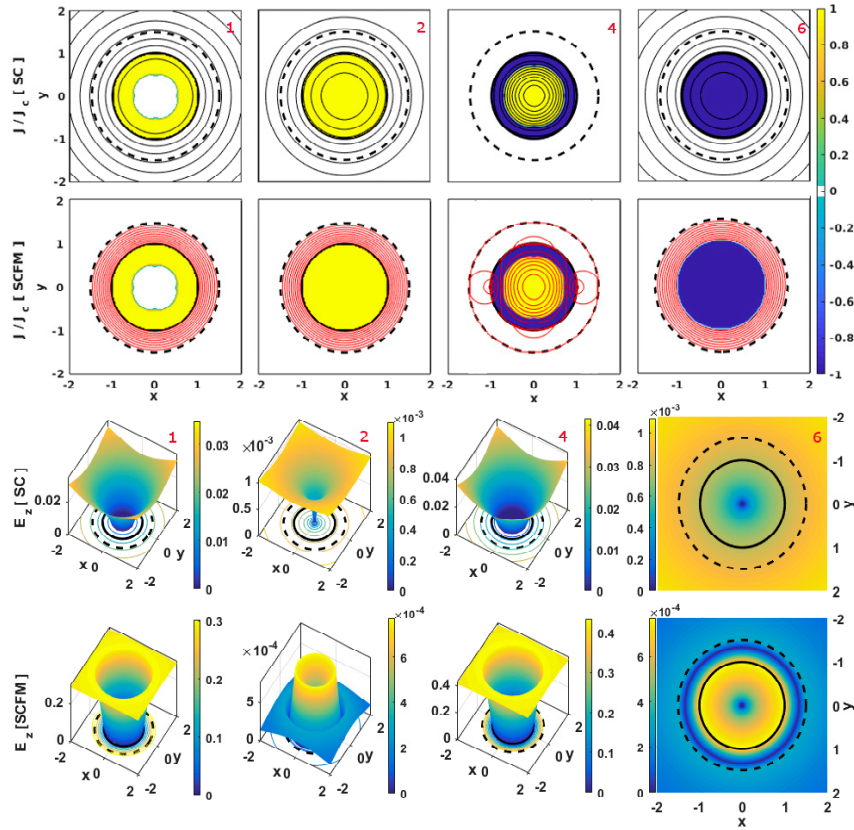

Figure 2. (Color online) Time dynamics of the current density profiles for the bare (1st row) and FM-sheathed SC heterostructures (2nd row) in accordance with the time-steps 1, 2, 4, and 6 in figure 1 (a) for $I_{a}=I_{c}$. Lighter (yellow) regions indicate positive current densities $J_{t r}=+J_{c}$, and darkest (blue) regions indicate $J_{i}=-J_{c}$. A set of maximum 10 contour lines of the total magnetic vector potential (see (5)-(7)) are displayed for illustration purposes. Analogously, the bottom two rows show corresponding electric field profiles for the SC and SCFM configurations, respectively. Electric field values are given in units of $\left(\mu_{0} / 4 \pi\right) R_{1}^{2} J_{c} / \delta_{t}$.

an electrical resistivity several orders of magnitude greater that the one of the SC, an assumption generally valid for all type-II SCs at operative temperatures below $T_{c}$ and low to moderate frequencies $(<1 \mathrm{MHz})[26]$. Therefore, if $\Omega$ can be divided into a mesh of elements containing a sufficiently large number of infinitely long domains, it is possible to reduce the problem to the calculation of the self and mutual inductance matrices, or vector potentials, for lines of current density $J_{i}$ located at the positions $r_{i} \in \Omega$, i.e., [34]

$$
\begin{gathered}
\mathbf{A}_{s}\left(\mathbf{r}_{i}\right)=\frac{\mu_{0}}{4 \pi} \pi \mathbf{J}_{i} \\
\mathbf{A}_{m}\left(\mathbf{r}_{i}\right)=-\frac{\mu_{0}}{4 \pi} \mathbf{J}_{i} \operatorname{Ln}\left(r_{i j^{2}}\right) \quad \text { for } \quad r_{i j} \neq 0
\end{gathered}
$$

with $r_{i j}$ the distance between two lines of current, each at the positions $r_{i}$ and $r_{j}$ respectively. This set of equations allows to reproduce all the electromagnetic properties of type-II SCs for diverse material laws, either material dependant $E-J$ power laws [36], or the pure CS law $|J| \leq\left|J_{c}\right|$ [28], the latter used in this study for the sake of generality. Nonetheless, in order to consider the magnetic component induced by the FM sheath by the action of the elements of current inside the SC, it is necessary to write these vector potentials into complex coordinates such that a multipole expansion can be invoked for determining the total magnetic vector potential at the three regions of interest (i) $r_{i} \leq R_{1}$ (within the SC), (ii) $R_{1}<r_{i} \leq R_{2}$ (within the FM), and (iii) $r_{i}>R_{2}$ (outside the SCFM heterostructure).
After a long series of analytical calculations, we have found that the contribution of the FM sheath to the magnetic vector potential over the SC domain can be written as

$$
\mathbf{A}_{F M}\left(\mathbf{r}_{j}<R_{1}\right)=-\frac{\mu_{0}}{4 \pi}\left(\mu_{r}^{2}-1\right) \mathbf{J}_{i} \times \Gamma_{S C},
$$

with $\Gamma_{S C}$ defined by

$$
\sum_{n=1}^{\infty}\left\{\frac{2}{n}\left(\frac{r_{i} r_{j}}{R_{1}^{2}}\right)^{n}\left[\frac{R_{2}^{2 n}-R_{1}^{2 n}}{R_{1}^{2 n}\left(\mu_{r}-1\right)^{2}-R_{2}^{2 n}\left(\mu_{r}+1\right)^{2}}\right] \cos \left(n \phi_{j}\right)\right\} .
$$

Thus, by imposing the CS law over the minimization functional in (4), and by having defined the different contributions to the magnetic vector potential within the SC domain, it is possible to find the full solution of the electromagnetic problem within $\Omega$, as it is shown in Section III

\section{EleCtromagnetic Features And AC Losses}

As it can be seen in the top row of figure 2, when the SC wire is in absence of the FM sheath, the flux front profiles of current density calculated by our numerical method follow the same circular patterns of radius $\widehat{r}=R_{1} \sqrt{\left(1-I_{t r} / I_{c}\right)}$ that can be obtained by exact analytical methods [37], with lines of magnetic field extending to all areas of space beyond $\widehat{r}$, as long as $I_{t r} \neq 0$, otherwise the field lines are confined within the SC domain $\left(r_{i}<R_{1}\right)$ (1st row, 3rd column at Fig. 2). For the sake of clarity, it is worth emphasizing that the 'field' lines shown in this figure are not the lines of magnetic field but the contour lines of the magnetic vector potential, which although both have the same symmetry, the later only disclose the points over a closed curve where the intensity of the magnetic field is the same. In this sense, the lack of contour lines in areas filled with current density as the ones observed at the time steps 2 and 6 , are not meant to be interpreted as zero-field regions. In fact, the only situation when there is no evidence of magnetic field within or beyond the domains of current, is when $I_{a}=0$. Nevertheless, as the scope of this paper focuses on the electric field and AC losses of SCFM heterostructures, a detailed discussion on how the resulting magnetic flux profiles are compared with the classical magnetic field profiles for rounded SC wires [38] is being prepared for a further publication. Thus, just for illustration purposes, what can be observed in the second row of Fig. 2, i,e., when the SC is covered by a FM sheath of radius $R_{2}>R_{1}$, is that the distribution of $J$-profiles is the same than the one attained for the SC barewire, but with the FM acting as a magnetic field trap, which consequently implies that the increment in the $\mathrm{AC}$ losses in a SCFM heterostructure can only be produced by the magneto coupling between these two materials, but not by an alteration of the critical current of the SC.

However, knowing the distribution of current inside the $\mathrm{SC}$ is not enough to determine the total electric field $\mathbf{E}=$ $-d \mathbf{A} / d t+C_{t}$ that is experienced by the different sections of the SCFM heterostructure, unless the contribution of the FM sheath to the vector potential is explicitly included. For instance, in the case of the bare $\mathrm{SC}$, the total vector potential is simply defined as $\mathbf{A}_{S C}\left(\mathbf{r}_{i} \leq R_{1}\right)=\mathbf{A}_{s}+\mathbf{A}_{m}$, whilst for the SCFM heterostructure it results to be $\mathbf{A}_{S C F M}\left(\mathbf{r}_{i} \leq\right.$ $\left.R_{1}\right)=\mathbf{A}_{s}+\mathbf{A}_{m}+\mathbf{A}_{F M}$, therefore increasing the magnitude 
of the electric field inside the SC, as it can be seen when comparing the electric field results displayed in figure 2 for the bare SC, $E_{z}[S C]$, and the SCFM heterostructure, $E_{z}[F M]$. Thus, for $d \mathbf{A} / d t \neq 0$, i.e., for $I_{t r} \neq \pm I_{a}$ (see results for time steps 1 and 4 in figure 2), we have found that for the SCFM heterostructure the electric field at the SC surface $\left(r_{i}=R_{1}\right)$ increases in at least one order of magnitude $\left(E_{z}\left[F M @ R_{1}, t_{1}\right]=0.01297\right)$ when compared with the sole SC $\left(E_{z}\left[S C @ R_{1}, t_{1}\right]=0.001104\right)$, both expressed in units of $\left(\mu_{0} / 4 \pi\right) R_{1}^{2} J_{c} / \delta_{t}$. However, at the outer surface of the SCFM heterostructure, and in fact beyond of it $\left(r_{i} \geq R_{2}\right)$, the effect on the electric field by the FM sheath is much more acute as it can be seen in figure 2, reaching up to more than two orders of difference in their magnitude, where $E_{z}\left[S C @ R_{2}, t_{1}\right]=$ 0.01866 , and $E_{z}\left[F M @ R_{2}, t_{1}\right]=0.3019$. On the other hand, for the current peak values, $I_{t r}= \pm I_{a}$ (time steps 2 and 6 , respectively), the change in the electric field at $r_{i}=R_{1}$ is negligible (as expected), or to be more precise, it is in the same order of magnitude of the allowed numerical tolerance $(1 E-6)$ within our computational model. However, for $\left(R_{1}<r_{i} \leq R_{2}\right)$ the FM sheath shows a fast lowering of the electric field leakage outside the heterostructure, but this only happens at the peak values of $I_{t r}$. Thus, for power cable applications, a remarkable feature of this study is that we have demonstrated that by the use of SCFM heterostructures, even in the most simple configurations as the one envisaged in this paper, certain cautions must be taken regarding the dielectric properties of the insulations due to the significant increase in the electric field that is being produced by the $\mathrm{AC}$ current and the SC/FM coupling. Therefore, although the benefits of the magnetic shielding properties of SCFM heterostructures are undeniable, a further challenge is created regarding the setting of adequate insulations for the prevention of electric breakdowns in these systems. Moreover, despite neither the electric field nor the distribution of superconducting currents at the peak values change between the bare SC and the SCFM heterostructure, and therefore no change in the instantaneous energy losses $\mathbf{E} \cdot \mathbf{J}$ at theses instants can be observed, it does not mean that the hysteresis losses also called AC losses does not change. In fact, as the calculation of the $\mathrm{AC}$ losses per unit time and volume $(\Phi)$ for cyclic excitations of frequency $\omega$ invokes the magnetic history of the SC cross-section $(\Omega)$ between the two peaks of the $I_{t r}$ excitation through the vector potential $\mathbf{A}_{S C F M}$,

$$
\frac{L}{\omega}=\oint_{t_{2}}^{t_{10}} d t \int_{\Phi} \mathbf{E} \cdot \mathbf{J} d \Phi=-2 \sum_{t_{2}}^{t_{6}} \partial \mathbf{A}_{S C F M} \cdot \mathbf{J}_{\Omega},
$$

the observed increment of the electric field then has a backlash onto the total calculation of the $\mathrm{AC}$ losses, even when an ideal soft ferromagnet with negligible magnetization losses is considered.

In figure 3, we show our numerical results for the AC losses of the bare SC which coincides with the analytical solution of Gurevich et al., [39], it compared with our calculated AC losses for the SCFM heterostructure. Therein, we can observe how the overall AC losses of the SCFM heterostructure increases due to the magnetostatic coupling between the SC

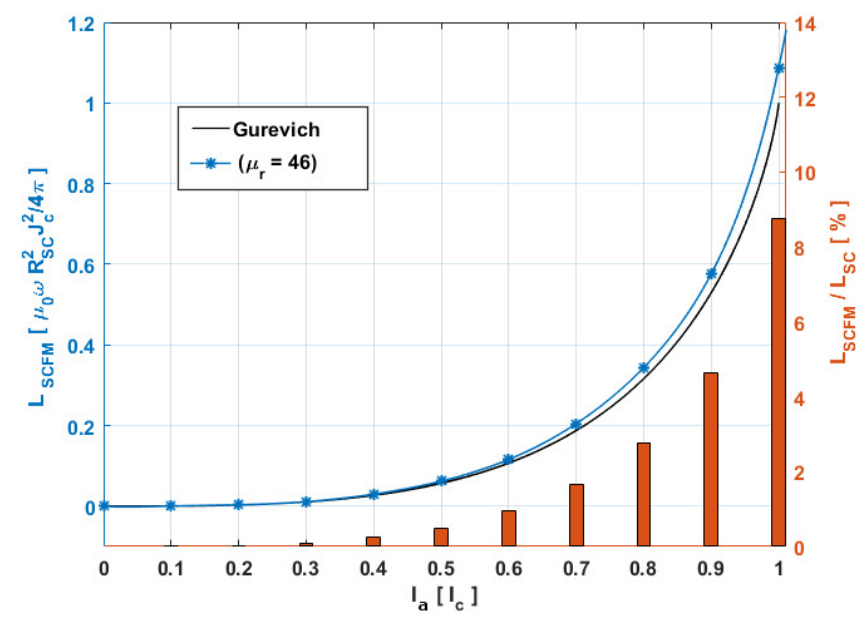

Figure 3. (Color Online) Hysteretic AC losses per cycle in units of $\left(\mu_{0} / 4 \pi\right) R_{1}^{2} J_{c}^{2}$ for the SCFM heterostructure (blue line with star symbols) depicted in figure 1 (c), it compared with the analytical approach of Gurevich et al., [39] for a bare rounded SC. Color bars show the percentage increment in the AC losses (right scale) when comparing the bare SC and the SCFM heterostructure.

and the FM as $I_{a}$ increases, reaching up to $\sim 9 \%$ greater losses when $I_{a}$ is as high as the critical current threshold $I_{c}$. Therefore, if any further increment in the AC losses of a SCFM cable of similar topology is experimentally obtained, this can be only caused by a non-negligible contribution of the magnetization loop in the FM, as long as adequate measures to subtract non-adiabatic and joints losses are considered.

\section{CONCLUding REMARKS}

In this paper, the electromagnetic response of a SCFM heterostructure composed by a type-II SC of cylindrical cross section and radius $R_{1}$, it embedded within an ideal soft ferromagnetic sheath (FM) of relative magnetic permeability $\mu_{r}=46$ and radius $R_{2}=1.5 R_{1}$, has been presented. Our model is based onto the variational formulation of the generalized CS model introduced in [28], together with an exact analytical calculation of the magnetostatic coupling between the SC and the FM in cylindrical topologies (figure 11 (c)). We have demonstrated that beyond the well known magnetic shielding advantages that FM sheaths add to hybrid SCFM heterostructures under external magnetic field conditions, when the SC is subjected only to an alternating current (self-field condition), a significant increment in the electric field over and beyond the heterostructure can be observed for the transient states in between the peak values of $I_{t r}$. This might imply significant repercussions in the designing of HV power cables aimed to utilize the properties of hybrid SC/FM structures, as further considerations must be taken regarding the dielectric properties of the insulating materials used.

Moreover, we have demonstrated the existence of an inherent coupling between the macroscopic current densities inside the SC and the magnetic inductance elements created by the FM, even when the latter has negligible magnetization losses. Therefore, due to this new physical mechanism which can be 
treated by means of multipolar expansions, the overall $\mathrm{AC}$ losses of the SCFM heterostructure increases in up to $9 \%$ for AC currents of $I_{a}=I_{c}$, an increment that cannot be ignored in practical applications. The electromagnetic phenomena shown in this paper can be used as a benchmark to understand the nature of increments in the AC losses for SCFM heterostructures, which cannot be explained by conventional mechanisms.

\section{REFERENCES}

[1] B. Glowacki and M. Majoros, "Superconducting-magnetic heterostructures: a method of decreasing ac losses and improving critical current density in multifilamentary conductors," Journal of Physics: Condensed Matter, vol. 21, no. 25, p. 254206, 2009.

[2] A. Sanchez, C. Navau, J. Prat-Camps, and D.-X. Chen, "Antimagnets: controlling magnetic fields with superconductor-metamaterial hybrids," New Journal of Physics, vol. 13, no. 9, p. 093034, 2011.

[3] C. Navau, J. Prat-Camps, O. Romero-Isart, J. I. Cirac, and A. Sanchez, "Long-distance transfer and routing of static magnetic fields," Physical review letters, vol. 112, no. 25, p. 253901, 2014.

[4] F. Gömöry, M. Solovyov, J. Souc, C. Navau, J. Prat-Camps, and A. Sanchez, "Experimental realization of a magnetic cloak," Science, vol. 335 , no. 6075, pp. 1466-1468, 2012.

[5] Y. A. Genenko, H. Rauh, and S. Kurdi, "Finite-element simulations of hysteretic alternating current losses in a magnetically coated superconducting tubular wire subject to an oscillating transverse magnetic field," Journal of Applied Physics, vol. 117, no. 24, p. 243909, 2015.

[6] F. Gömöry, M. Solovyov, and J. Šouc, "Magnetization loop modelling for superconducting/ferromagnetic tube of an ac magnetic cloak," Superconductor Science and Technology, vol. 28, no. 4, p. 044001, 2015.

[7] J. Šouc, M. Solovyov, and F. Gömöry, "Hiding objects in ac magnetic fields of power grid frequency by two-shell ferromagnetic/superconducting cloak," Applied Physics Letters, vol. 109, no. 3, p. 033507, 2016.

[8] J. P. na Roche, Y. A. Genenko, and A. Badía-Majós, "Magnetic invisibility of the magnetically coated type-ii superconductor in partially penetrated state," Applied Physics Letters, vol. 109, no. 9, p. 092601, 2016.

[9] M. Baghdadi, H. S. Ruiz, and T. A. Coombs, "Nature of the low magnetization decay on stacks of second generation superconducting tapes under crossed and rotating magnetic field experiments," Scientific Reports, vol. 8, no. 1, p. 1342, 2018.

[10] Y. A. Genenko, S. V. Yampolskii, and A. V. Pan, "Virgin magnetization of a magnetically shielded superconductor wire: Theory and experiment," Applied Physics Letters, vol. 84, no. 19, pp. 3921-3923, 2004.

[11] M. Solovyov, J. Šouc, and F. Gömöry, "Magnetic cloak for low frequency ac magnetic field," IEEE Transactions on Applied Superconductivity, vol. 25, no. 3, pp. 1-5, June 2015.

[12] C. Pang, A. Campbell, and P. McLaren, "Losses in nb/ti multifilamentary composite when exposed to transverse alternating and rotating fields," IEEE Transactions on Magnetics, vol. 17, no. 1, pp. 134-137, Jan 1981

[13] H. R. Kirchmayr, "Permanent magnets and hard magnetic materials," Journal of Physics D: Applied Physics, vol. 29, no. 11, p. 2763, 1996.

[14] M. Itoh, K. Mori, and T. Minemoto, "Magnetic shielding effects by the superposition of a six-layered ferromagnetic cylinder over a bpscco cylinder," IEEE Transactions on Magnetics, vol. 32, no. 4, pp. 2605$2608,1996$.

[15] G. P. Lousberg, J. Fagnard, M. Ausloos, P. Vanderbemden, and B. Vanderheyden, "Numerical study of the shielding properties of macroscopic hybrid ferromagnetic/superconductor hollow cylinders," IEEE Transactions on Applied Superconductivity, vol. 20, no. 1, pp. 33-41, Feb 2010.

[16] J. Horvat, W. K. Yeoh, J. H. Kim, and S. X. Dou, "Transport and magnetic critical current in superconducting mgb 2 wires," Superconductor Science and Technology, vol. 21, no. 6, p. 065003, 2008.

[17] J. Horvat, S. Soltanian, and W. K. Yeoh, "The relevance of the self-field for the 'peak effect' in the transport $\mathrm{j} \mathrm{c}(\mathrm{h})$ of iron-sheathed mgb 2 wires," Superconductor Science and Technology, vol. 18, no. 5, p. 682, 2005.

[18] P. Kováč, I. Hušek, T. Melišek, M. Kulich, and V. Štrbík, "Mgb2 composite wires with fe, $\mathrm{nb}$ and ta sheaths," Superconductor Science and Technology, vol. 19, no. 6, p. 600, 2006.
[19] G. P. Lousberg, J.-F. Fagnard, X. Chaud, M. Ausloos, P. Vanderbemden, and B. Vanderheyden, "Magnetic properties of drilled bulk high-temperature superconductors filled with a ferromagnetic powder," Superconductor Science and Technology, vol. 24, no. 3, p. 035008, 2011.

[20] L. Gozzelino, R. Gerbaldo, G. Ghigo, F. Laviano, A. Agostino, E. Bonometti, M. Chiampi, A. Manzin, and L. Zilberti, "Dc shielding properties of coaxialMgB $\mathrm{Mg}_{2} /$ Fecups," IEEE Transactions on Applied Superconductivity, vol. 23, no. 3, pp. 8201 305-8 201305 , June 2013.

[21] L. Gozzelino, R. Gerbaldo, G. Ghigo, F. Laviano, and M. Truccato, "Comparison of the shielding properties of superconducting and superconducting/ferromagnetic bi- and multi-layer systems," Journal of Superconductivity and Novel Magnetism, vol. 30, no. 3, pp. 749-756, Mar 2017.

[22] Y. A. Genenko, H. Rauh, and S. V. Yampolskii, "The bean-livingston barrier at a superconductor/magnet interface," Journal of Physics: Condensed Matter, vol. 17, no. 10, p. L93, 2005.

[23] O. Tsukamoto, H. Nakayama, S. Odaka, M. Ciszek, S. Hahakura, M. Ueyama, K. Ohmatsu, and D. Miyagi, "Transport current losses in hobacuo-123 coated conductors with a ni-alloy substrate," Physica C: Superconductivity and its Applications, vol. 426-431, pp. 1290 - 1294, 2005, proceedings of the 17th International Symposium on Superconductivity (ISS 2004).

[24] M. Vojenčiak, J. Šouc, and F. Gömöry, "Critical current and ac loss analysis of a superconducting power transmission cable with ferromagnetic diverters," Superconductor Science and Technology, vol. 24, no. 7, p. 075001, 2011.

[25] P. Kováč, I. Hušek, T. Melišek, M. Ahoranta, J. Šouc, J. Lehtonen, and F. Gömöry, "Magnetic interaction of an iron sheath with a superconductor," Superconductor Science and Technology, vol. 16, no. 10, p. 1195, 2003.

[26] F. Fiorillo and C. Beatrice, "Energy losses in soft magnets from dc to radiofrequencies: Theory and experiment," Journal of Superconductivity and Novel Magnetism, vol. 24, no. 1, pp. 559-566, Jan 2011.

[27] Y. Mawatari, "Magnetic field distributions around superconducting strips on ferromagnetic substrates," Phys. Rev. B, vol. 77, p. 104505, Mar 2008.

[28] A. Badía-Majós, C. López, and H. S. Ruiz, "General critical states in type-II superconductors," Phys. Rev. B, vol. 80, p. 144509, Oct 2009.

[29] Y. A. Genenko, H. Rauh, and P. Krüger, "Finite-element simulations of hysteretic ac losses in a bilayer superconductor/ferromagnet heterostructure subject to an oscillating transverse magnetic field," Applied Physics Letters, vol. 98, no. 15, p. 152508, 2011.

[30] S. V. Yampolskii, Y. A. Genenko, H. Rauh, and A. V. Snezhko, "The bean model of the critical state in a magnetically shielded superconductor filament," Journal of Physics: Conference Series, vol. 43, no. 1, p. $576,2006$.

[31] H. S. Ruiz, C. López, and A. Badía-Majós, "Inversion mechanism for the transport current in type-II superconductors," Phys. Rev. B, vol. 83, p. 014506, Jan 2011.

[32] F. Gömöry, R. Tebano, A. Sanchez, E. Pardo, C. Navau, I. Husek, F. Strycek, and P. Kovac, "Current profiles and ac losses of a superconducting strip with an elliptic cross-section in a perpendicular magnetic field," Superconductor Science and Technology, vol. 15, no. 9, p. 1311, 2002.

[33] G. P. Mikitik, Y. Mawatari, A. T. S. Wan, and F. Sirois, "Analytical methods and formulas for modeling high temperature superconductors," IEEE Transactions on Applied Superconductivity, vol. 23, no. 2, pp. 8001 920-8 001920 , April 2013.

[34] H. S. Ruiz, A. Badía-Majós, Y. A. Genenko, H. Rauh, and S. V. Yampolskii, "Superconducting wire subject to synchronous oscillating excitations: Power dissipation, magnetic response, and low-pass filtering," Applied Physics Letters, vol. 100, no. 11, p. 112602, 2012.

[35] H. S. Ruiz and A. Badía-Majós, "Exotic magnetic response of superconducting wires subject to synchronous and asynchronous oscillating excitations," Journal of Applied Physics, vol. 113, no. 19, p. 193906, 2013.

[36] A. Badía-Majós and C. López, "Modelling current voltage characteristics of practical superconductors," Superconductor Science and Technology, vol. 28 , no. 2, p. $024003,2015$.

[37] H. S. Ruiz, A. Badía-Majós, Y. A. Genenko, and S. V. Yampolskii, "Strong localization of the density of power losses in type-II superconducting wires," IEEE Transactions on Applied Superconductivity, vol. 23, no. 3, pp. 8000404-8000 404, June 2013.

[38] H. S. Ruiz, Material laws and numerical methods in applied superconductivity. Zaragoza, Spain: University of Zaragoza Press, 2013.

[39] A. V. Gurevich, R. G. Mints, and A. L. Rakhmanov, Physics of Composite Superconductors. Begell House, New York, 1997. 